\title{
Novel and Improved Method for the Synthesis of 2-mercaptobenzimidazole Derivatives
}

\author{
A. EL KIHEL ${ }^{1 *}$, H. AIT SIR ${ }^{1}$, S. JEBBARI ${ }^{1}$, M. AHBALA ${ }^{1}$, S. GUESMI ${ }^{2}$ and P. BAUCHAT ${ }^{3}$ \\ 'Laboratoire de chimie bioorganique, Faculté des Sciences, BP20, 24000, El Jadida, Maroc. \\ ${ }^{2}$ Laboratoire de Chimie de Coordination et d'Analytique, Faculté des Sciences, \\ BP20, 24000, El Jadida, Maroc. \\ ${ }^{3}$ Université de Rennes1, Sciences Chimiques de Rennes U.M.R.6226, ICMV, Campus de Beaulieu, \\ Avenue Général Leclerc, 35042, Rennes, France \\ *Corresponding author E-mail: abdellatifelkihel@gmail.com \\ http://dx.doi.org/10.13005/ojc/320405
}

(Received: July 10, 2016; Accepted: August 11, 2016)

\begin{abstract}
2-mercaptobenzimidazole derivatives were synthesized by reaction of o-phenylenediamines with $\mathrm{N}$-aminorhodanine. This reaction represent a new synthesis of 2-mercaptobenzazole. The structure of the obtained products was established by spectroscopic data.
\end{abstract}

Keywords: 2-mercaptobenzimidazole, o-phenylenediamines, $\mathrm{N}$-aminorhodanine.

\section{INTRODUCTION}

Thiols are very useful building blocks for the synthesis of various organosulfur compounds: they have several applications in organic synthesis, in bioorganic, medicinal and heterocyclic chemistry". Also, in addition to that, thiols can act as safety-catch linker in peptide chemistry². Morever, thiols have been employed as sulfur-based ligands in transition metal complexes $^{3,4}$. In this respect, a number of synthetic methods for the preparation of thiol derivatives by the reaction of o-phenylendiamine with carbon disulphide $^{5,9}$, thiourea ${ }^{10,11}$, O-isopropylxanthic acid potassium salt ${ }^{12}, 5$-phenyl-1,3,4-oxadiazole-2(3H)- thione derivatives and $\mathrm{N}$-phenylisothiocyanate ${ }^{13}$, thiocyanic acid ammoniac salt ${ }^{14}$. In continuation of our research concerning benzimidazoles ${ }^{15}, 19$. The condensation of o-phenylenediamine with $\mathrm{N}$-aminorhodanine was carried out.

\section{Experimental section \\ General Procedure}

O-phenylenediamines $(0.065 \mathrm{~mol})$ was heated with $\mathrm{N}$-aminorhodanine $(0.065 \mathrm{~mol})$ in xylene $(50 \mathrm{ml})$ for 5 hours. The obtained residue was filtered and was crystallized from aqueous alcohol (charcoal). The obtained solid was recrystallized in ethanol. 


\section{2-mercaptobenzimidazole $3 a$}

Yield $=87 \% ; \mathrm{mp}>250^{\circ} \mathrm{C} .1 \mathrm{HNMR}$ (DMSO-d6): 7.10 (m, 2Har); 7.27 (m, 2Har); 12.42 (s,NH).13CNMR (DMSO-d6): $119.43(\mathrm{CH}) ; 126.36$ (CH); $138.82(\mathrm{C}) ; 167.12(\mathrm{C}=\mathrm{S})$. HRMS, m/z: 150(M), calcd for $\mathrm{C}_{7} \mathrm{H}_{6} \mathrm{~N}_{2} \mathrm{~S}: 150.02517$, found: 150.0251 .

\section{2-mercapto-5-nitrobenzimidazole $3 \mathrm{~b}$}

Yield $=81 \% ; \mathrm{mp}>250^{\circ} \mathrm{C}$. $1 \mathrm{HNMR}$ (DMSO$\mathrm{d} 6): 7.12(\mathrm{~d}, \mathrm{JAB}=8.3 \mathrm{~Hz}, 1 \mathrm{Har}) ; 7.29(\mathrm{q}, \mathrm{JBX}=2.1 \mathrm{~Hz}$, $\mathrm{JAB}=8.3 \mathrm{~Hz}, 1 \mathrm{Har}) ; 7.46(\mathrm{~d}, \mathrm{JBX}=2.1 \mathrm{~Hz}, 1 \mathrm{Har})$; 12.03 (s, NH).13CNMR (DMSO-d6):108.24 (CH); $113.46(\mathrm{CH}) ; 125.70(\mathrm{CH}) ; 137.45(\mathrm{C}) ; 141.94(\mathrm{C}) ;$ $148.01(\mathrm{C}) ; 167.54(\mathrm{C}=\mathrm{S}) . \mathrm{HRMS}, \mathrm{m} / \mathrm{z}:$ 195(M), calcd for $\mathrm{C}_{7} \mathrm{H}_{5} \mathrm{~N}_{3} \mathrm{O}_{2} \mathrm{~S}: 195.01025$, found: 195.0102 .

\section{2-mercapto-5-methylbenzimidazole 3c}

Yield $=83 \% ; \mathrm{mp}>250^{\circ} \mathrm{C}$. ${ }^{1} \mathrm{HNMR}$ (DMSO$\left.\mathrm{d}_{6}\right): 2.21\left(\mathrm{~s}, \mathrm{CH}_{3}\right) ; 6.43(\mathrm{~d}, \mathrm{JAB}=8.3 \mathrm{~Hz}, 1 \mathrm{Har}) ; 6.83$ (q, JBX=1.7Hz, JAB= 8.3Hz, 1Har); 7.05 (d, JBX= 1.7Hz, $1 \mathrm{Har}$ ); 12.03 (s, NH). ${ }^{13} \mathrm{CNMR}$ (DMSO- $\mathrm{d}_{6}$ ):
$21.31\left(\mathrm{CH}_{3}\right) ; 109.50(\mathrm{CH}) ; 109.99(\mathrm{CH}) ; 123.49(\mathrm{CH})$; $130.69(\mathrm{C}) ; 131.92(\mathrm{C}) ; 132.95(\mathrm{C}) ; 168.12(\mathrm{C}=\mathrm{S})$. HRMS, m/z: $164(\mathrm{M})$, calcd for $\mathrm{C}_{9} \mathrm{H}_{8} \mathrm{~N}_{2} \mathrm{~S}: 164.04082$, found: 164.0408 .

\section{5-chloro-2-mercaptobenzimidazole 3d}

Yield $=79 \% ; \mathrm{mp}>250^{\circ} \mathrm{C}$. $1 \mathrm{HNMR}(\mathrm{DMSO}$ d6): 6.81 (d, JAB= 7.9Hz, $1 \mathrm{Har}$ ); 7.03 (q, JBX=1.9Hz, $\mathrm{JAB}=7.9 \mathrm{~Hz}, 1 \mathrm{Har}) ; 7.25(\mathrm{~d}, \mathrm{JBX}=1.9 \mathrm{~Hz}, 1 \mathrm{Har})$ 11.83 (s, NH).13CNMR (DMSO-d6):101.34 (CH); $109.64(\mathrm{CH}) ; 123.71(\mathrm{CH}) ; 135.54(\mathrm{C}) ; 140.84(\mathrm{C})$; $146.14(\mathrm{C}) ; 165.24(\mathrm{C}=\mathrm{S})$. HRMS, m/z: 183(M), calcd for $\mathrm{C}_{7} \mathrm{H}_{5} \mathrm{CIN}_{2} \mathrm{~S}: 183.9962$, found: 183.996 .

\section{5,6-dichloro-2-mercaptobenzimidazole 3e} Yield $=75 \%$; $\mathrm{mp}>250^{\circ} \mathrm{C}$. ${ }^{1} \mathrm{HNMR}(\mathrm{DMSO}$ $\left.\mathrm{d}_{6}\right): 7.79$ (s, 1Har); $11.82(\mathrm{~s}, \mathrm{NH}) \cdot{ }^{13} \mathrm{CNMR}\left(\mathrm{DMSO}-\mathrm{d}_{6}\right)$ : $128.60(\mathrm{CH}) ; 127.25(\mathrm{C}) ; 135.49(\mathrm{C}) ; 168.30$ (C=S). HRMS, m/z: 217(M), calcd for $\mathrm{C}_{7} \mathrm{H}_{4} \mathrm{~N}_{2} \mathrm{~S}: 217.94722$, found: 217.9472<smiles>[R]c1cc(N)c(N)cc1[R]</smiles>

1a-f $\mathrm{R}=\mathrm{H}, \mathrm{CH}_{3}, \mathrm{Cl}, \mathrm{NO}_{2}$ $\mathbf{R}^{\prime}=\mathbf{H}, \mathrm{CH}_{3}, \mathbf{C L}$<smiles></smiles>

Xylene<smiles>[R]c1cc2[nH]c(=S)[nH]c2cc1[R]</smiles>

2a-f

$\mathrm{R}=\mathrm{H}, \mathrm{CH}_{3}, \mathrm{Cl}, \mathrm{NO}_{2}$ $\mathrm{R}^{\prime}=\mathrm{H}, \mathrm{CH}_{3}, \mathrm{CL}$

Scheme 1: The reaction of 2-mercaptobenzimidazoles synthesis<smiles>CC(C)CCCCCCCC(N)=S=S</smiles>

Scheme 2: Proposed formation mechanism of the product 


\section{5,6-dimethyl-2-mercaptobenzimidazole $3 f$}

Yield $=80 \%$; $\mathrm{mp}>250^{\circ} \mathrm{C}$. ${ }^{1} \mathrm{HNMR}$ (DMSO$\left.\mathrm{d}_{6}\right): 2.32\left(\mathrm{~s}, \mathrm{CH}_{3}\right) ; 7.02$ (s, 1Har); 12.63 (s, NH). ${ }^{13}$ CNMR (DMSO-d $)$ : $20.43\left(\mathrm{CH}_{3}\right) ; 111.00(\mathrm{CH})$; $131.25(\mathrm{C}) ; 131.59(\mathrm{C}) ; 168.00(\mathrm{C}=\mathrm{S})$. HRMS, m/z: 178(M), calcd for $\mathrm{C}_{9} \mathrm{H}_{10} \mathrm{~N}_{3} \mathrm{~S}: 178.05647$, found: 178.0564 .

\section{RESULTS AND DISCUSSION}

In this work, we report a novel method for synthesis of 2-mercaptobenzimidazole derivatives. 2- Mercaptobenzimidazoles are interesting starting compounds because of their chemical reactivity and biological activities. A mixture of o-phenylenediamines $1(\mathrm{a}-\mathrm{g})$ and $\mathrm{N}$-aminorhodanine in xylene was heated during 8 hours. (Scheme 1). The structure of the products $2(\mathrm{a}-\mathrm{g})$ has been determined by NMR and mass data. 1HNMR spectra showed the presence of the signal of $\mathrm{NH}$ and the signal of the $\mathrm{C}=\mathrm{S}$ was observed in 13CNMR spectra which confirms the structure of the products $2(\mathrm{a}-\mathrm{g})$.
A possible mechanism for the formation of the 2-mercaptobenzimidazole (2a-g) is shown in scheme 2. The key step is at the intermediate $A$, in which the cyclization goes to the $C=S$ group not to $\mathrm{C}=\mathrm{O}$ group in order to lead to the benzotriazepine product. In the reaction, the only 2-mercaptobenzimidazole product was obtained (Scheme 2)

\section{CONCLUSION}

In this work, we developed a novel and improved method for 2-mercaptobenzimidazole derivatives by the condensation of o-phenylenediamines with $\mathrm{N}$-aminorhodanine

\section{AKNOWLEDGEMENTS}

This work has been supported by the Chouaib Doukkali University

\section{REFERENCES}

1. McReynolds, M.D.; Doughtery, J.M.; Hanson, P.R. Chem. Rev. 2004, 104, 2239 - 2258.

2. Hwang, J.Y.; Gong, Y.D. J. Comb. Chem. 2006, 8, 297 - 303.

3. Goh, L.Y.; Teo, M.E.; Khoo, S.B.; Leong, W.K.; Vittal, J.J. J. Organomet. Chem. 2002, 664, $161-169$.

4. Christensen, C.A.; Meldal, M. J. Comb. Chem. 2007, 9, 79 - 85.

5. Pae, A.N.; MIN, S. J. ; Roh, E. J.; YANG, H.Y.; Kim, T. H.; PARK, B. G.; Cho, Y. S. U.S. Pat. Appl. Publ. 20140114067, 24 Apr 2014

6. Rivera, A.; Maldonado, M.; Rios-Motta, J. Molecules 2012, 17, 8578 - 8586.

7. Maske, P.P.; Lokapure, S.G.; Nimbalkar, D.; Disouza, J.I. Pharma Chemica 2012, 4(3), 1283 - 1287.

8. Zhivotova, T.S.; Gazaliev, A.M.; Fazylov, S.D.; Aitpaeva, Z.K.; Turdybekov, D.M. Russian Journal of Organic Chemistry 2006, 42(3), $448-450$.

9. Valdez, J.; Cedillo, R.; Hernández-Campos, A.;Yépez, L.; Hernández-Luis, F.; NavarreteVázquez, G.; Tapia, A.; Cortés, R.; Hernández,
M.; Castillo, R. Bioorganic and Medicinal Chemistry Letters 2002, 12(16), 2221 2224.

10. Kidwai, M.; Saxena, S.; Mohan, R. Journal of the Korean Chemical Society 2005, 49(3), $288-291$.

11. Li, X. Youji Huaxue 2009, 29(7), 1129 1132.

12. Kumar, N.D.M.; Dubey, P.K. Indian Journal of Chemistry, Section B: Organic Chemistry Including Medicinal Chemistry 2012 , 51B(11), 1619 - 1622.

13. Khajavi, M.S.; Hajihadi, M.; Nikpour, F. Journal of Chemical Research, Synopses 1996, 2, $94-95$

14. Thakuria, H.; Das, G. ARKIVOC 2008, 15, $321-328$.

15. El kihel, A.; Zouitina, S.; Guesmi, S.; Ahbala, M.; Bauchat, P.; Biersack, B. Asian Journal of Chemistry 2016, 28(6), 1267 - 1269.

16. El kihel, A.; Ahbala, M.; Ramdane, H.; Nouiri, M.; Bauchat, P. International Journal of Development Research 2016, 6, 7295 $-7298$. 
17. El kihel, A.; Essassi, E.M.; Bauchat, P. Arabian Journal of Chemistry 2012, 5, 523 - 526

18. El kihel, A.; El ouar, M.; Ahbala, M.; Mouzdahir, A.; Harjane, T.; Knouzi, N. Arabian Journal of Chemistry 2010, 3, $9-12$
19. El kihel, A.; Ahbala, M.; Mouzdahir, A. ; Snader, N. ; Essassi, E.M.; Bauchat, P. ; Danion-Bougot, R. Synthetic Communications 2008, 38, 201 - 205. 